\title{
Dry micro-electro-discharge machining of carbon-nanotube forests
}

\section{using sulphur-hexafluoride}

\author{
Tanveer Saleh, Masoud Dahmardeh, Alireza Nojeh, Kenichi Takahata* \\ Department of Electrical and Computer Engineering, University of British Columbia, Vancouver \\ BC, V6T 1Z4, Canada
}

\begin{abstract}
The effect of using sulphur hexafluoride $\left(\mathrm{SF}_{6}\right)$, a high-dielectric-strength gas, for dry microelectro-discharge machining $(\mu \mathrm{EDM})$ of carbon-nanotube $(\mathrm{CNT})$ forests is investigated. It is found that $\mathrm{SF}_{6}$ enables $\mu \mathrm{EDM}$ of CNTs without $\mathrm{O}_{2}$, which is known to be essential for CNT machining in $\mathrm{N}_{2}$. The process in the $\mathrm{SF}_{6}$ ambient is found to lead to a smaller discharge gap, i.e., tighter tolerance as well as higher machining quality at a discharge voltage level (25 V) compared with the case of $\mathrm{N}_{2}$ at the same voltage. $\mathrm{N}_{2}$ environment produces lower discharge gap when a voltage level of $10 \mathrm{~V}$ is used. However, the quality of the machining (e.g. the inner post sidewall) seems to be somewhat poorer in this case, and rate of machining is somewhat lower. The mixture with $20 \% \mathrm{O}_{2}$ in $\mathrm{SF}_{6}$ is revealed to be an optimum condition in terms of both machining tolerance and quality. CNT forests are used as the cathode in the $\mathrm{SF}_{6}$-based $\mu \mathrm{EDM}$ process, as opposed to conventional $\mu \mathrm{EDM}$ where the workpiece forms the anode. This reversepolarity configuration in the $\mathrm{SF}_{6}-\mathrm{O}_{2}$ mixture is observed to generate higher discharge currents at low voltages, presumably due to effective field-emission by the CNTs, leading to finer and cleaner machining than the conventional normal-polarity case. Energy-dispersive X-ray analysis
\end{abstract}

\footnotetext{
* Corresponding author. Fax: +1-604-822-5949. Email: takahata@ece.ubc.ca
} 
reveals that the optimal machining conditions result in less contamination by the electrode element on the processed forest surfaces.

\section{Introduction}

Carbon nanotubes (CNTs) have highly attractive mechanical, electrical, optical, and thermal properties [1-5]. Densely packed, vertically aligned CNTs, commonly referred to as CNT forests, have many potential uses in different engineering fields as summarized in [6]. Patterning of CNT forests is crucial to make them useful for various applications. Selective growth of the forests by chemical vapor deposition (CVD) on pre-patterned catalyst on the substrate is widely used to produce two-dimensionally patterned CNT forests with uniform height. Recently, a microelectro-discharge machining $(\mu \mathrm{EDM})$ based process has been developed to enable threedimensional, free-form patterning of microstructures in bare CNT forests [7-10]. This process used dry air as the dielectric medium, instead of the dielectric liquid used in typical $\mu \mathrm{EDM}$, as the forest structures patterned in liquid are drastically modified when the structures are dried because of capillary effects [7]. This technique was further studied to investigate the possible removal mechanism of CNT forests in dry $\mu \mathrm{EDM}$, suggesting that the process was essentially oxygen plasma etching rather than the conventional, direct thermal removal (evaporation and melting) process, and that air (or $\mathrm{N}_{2}$ with $\sim 20 \% \mathrm{O}_{2}$ ) was an optimal medium for $\mu \mathrm{EDM}$ of CNT forests $[8,9]$. The machining tolerance, or the discharge gap clearance between a forest and the $\mu \mathrm{EDM}$ electrode, was reported to be $10 \mu \mathrm{m}$ or more, substantially larger than typical values (of one to a few $\mu \mathrm{m}$ ) involved in standard $\mu \mathrm{EDM}$ with dielectric liquid. In typical EDM (including $\mu \mathrm{EDM})$, the workpiece and the electrode are generally arranged to be the anode and the cathode, respectively, as this polarity usually results in efficient material removal with small electrode 
wear. Most of the previous studies on $\mu \mathrm{EDM}$ of CNT forests [7-9], as well as of carbon nanofibers [11], also used this conventional polarity, i.e., the carbon material served as the anode. Because of their extremely small tips with nanometer radii and high aspect ratios, CNTs are known to have excellent electron field-emission properties compared to the tungsten tip (a typical material used as $\mu \mathrm{EDM}$ tool) [12]. It has recently been found that reverse-polarity $\mu \mathrm{EDM}$ of CNT forests that are defined as the cathode in air ambient enhances the patterning tolerances and quality with decreased discharge energies [6]. In addition, all these previous studies have used the mixture of $\mathrm{N}_{2}$ and $\mathrm{O}_{2}$ (in the form of air in most cases) as the machining medium, and the effect of other gases on the $\mu \mathrm{EDM}$ performance has not been investigated so far. Sulphur hexafluoride $\left(\mathrm{SF}_{6}\right)$ has a dielectric strength much higher than that of $\mathrm{N}_{2}$ (by a factor of $\sim 3$ ) [13]. This suggests that in $\mathrm{SF}_{6}$, the machining electrode and the workpiece (CNT forest) have to come closer in order to cause a gas breakdown for a given electric field strength. Hence, the use of $\mathrm{SF}_{6}$ may further reduce the discharge gap in the $\mu \mathrm{EDM}$ process compared to the case with $\mathrm{N}_{2}$ under the same discharge voltage.

The present work investigates $\mu \mathrm{EDM}$ of pure CNT forests using a new gas, $\mathrm{SF}_{6}$. In the previous studies, it was reported that $\mathrm{O}_{2}$ was necessary for successful $\mu \mathrm{EDM}$ of CNT forest in a $\mathrm{N}_{2}$ environment [8,9]. This is unknown for an $\mathrm{SF}_{6}$ environment, which is experimentally investigated as part of this work. The study also explores the effect of $\mathrm{O}_{2}$ mixed in $\mathrm{SF}_{6}$ on the CNT removal process, and its comparison with the conventional $\mathrm{N}_{2}-\mathrm{O}_{2}$ environment. The characteristics of normal- and reverse-polarity $\mu \mathrm{EDM}$ for CNT forests in the $\mathrm{SF}_{6}-\mathrm{O}_{2}$ gas system and the patterned structures are studied to reveal various advantages over the conventional process in air ambient. 
2. Sample preparation and experimental set-up

The CNT forest samples used in this study were grown on highly doped silicon substrates (<100> n-type, resistivity $0.008-0.015 \Omega \cdot \mathrm{cm})$ using an ethylene-based atmospheric-pressure CVD system and iron catalyst. Details of the growth condition and system used for the sample preparation can be found in [6]. The process yielded forests of vertically aligned multi-walled

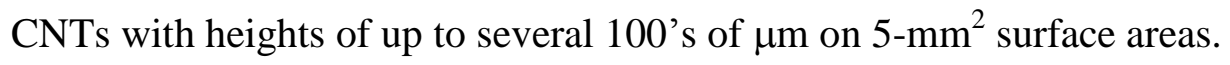

The experiments for $\mu \mathrm{EDM}$ of the CNT forests were performed using a servo-controlled 3axis $\mu$ EDM system (EM203, SmalTec International, IL, USA) with a $0.1-\mu \mathrm{m}$ positioning resolution. The discharge pulses were generated with a relaxation-type resistor-capacitor circuit [14], a proven pulse generator used for $\mu \mathrm{EDM}$ of CNTs [7-9]. The machine is capable of detecting undesired short-circuit events during operation and can retrieve the electrode held on the Z-axis stage upward to overcome a short circuit; once the short circuit is cleared, the electrode feeding is automatically resumed. The experimental set-up arranged for the machining tests and characterization is illustrated in Fig. 1. As shown, a current probe (CT-1, Tektronix, OR, USA) was used to monitor pulses of the discharge current in real time. The discharge current data were captured from the oscilloscope using a GPIB interface and stored in a computer for subsequent analysis. Further, $\mathrm{O}_{2}$ was first mixed with either $\mathrm{SF}_{6}$ or $\mathrm{N}_{2}$ inside a buffer chamber, and the mixed gas was introduced to the machining chamber, where the $\mathrm{O}_{2}$ concentration was 


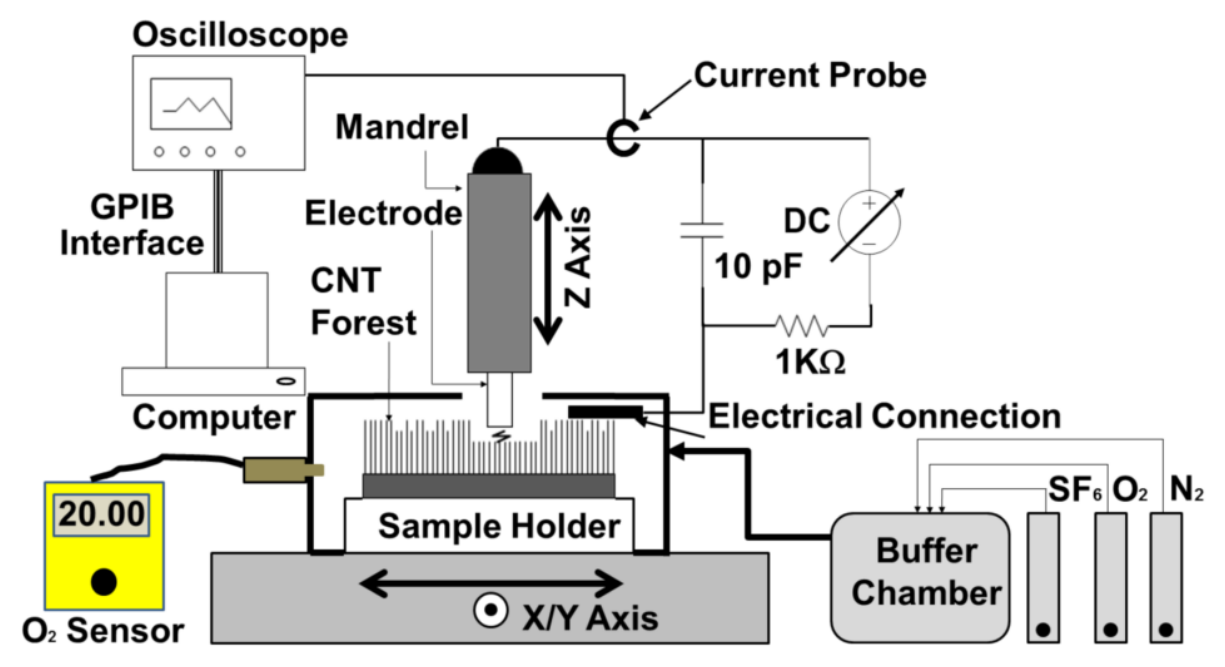

Fig. 1 - Experimental setup for dry $\mu \mathrm{EDM}$ of pure CNT forest in different gas media.

measured using an oxygen sensor (VN202, Vandagraph Co., UK). The flow rates of $\mathrm{O}_{2}$ and $\mathrm{SF}_{6} / \mathrm{N}_{2}$ were adjusted so that the $\mathrm{O}_{2}$ concentration reached the target value and was stabilized in the machining chamber for at least 5 minutes prior to machining. In this study, a series of $\mu$ EDM experiments at both the normal and reverse polarities were performed using cylindrical tungsten electrodes rotating at $3000 \mathrm{rpm}$. The $\mu \mathrm{EDM}$ conditions are summarized in Table 1 . A 10-pF capacitor was used in the discharge circuit for all the experiments in this study.

Table $1-\mu \mathrm{EDM}$ conditions used for CNT machining experiments.

\begin{tabular}{|c|c|c|}
\hline \multicolumn{2}{|c|}{ Parameters } & Values used \\
\hline \multicolumn{2}{|c|}{ Machining voltage $(\mathrm{V})$} & 25 or 10 \\
\hline Capacitance $(\mathrm{pF})$ & 10 \\
\hline \multicolumn{2}{|c|}{ Electrode feed rate during EDM $(\mathrm{mm} / \mathrm{min})$} & $\mathrm{Z}: 0.03, \mathrm{X}-\mathrm{Y}: 1$ \\
\hline \multirow{2}{*}{ Ambient } & $\mathrm{O}_{2} \%$ in $\mathrm{SF}_{6}\left(\right.$ for the $\mathrm{SF}_{6}-\mathrm{O}_{2}$ system) & $50,20,10$, or 0 \\
\cline { 2 - 3 } & $\mathrm{O}_{2} \%$ in $\mathrm{N}_{2}$ (for the $\mathrm{N}_{2}-\mathrm{O}_{2}$ system) & 20 or 0 \\
\hline
\end{tabular}




\section{Results and discussion}

As mentioned earlier, in air, $\mu \mathrm{EDM}$ of CNT forests with the reverse polarity using the forests as the cathode was demonstrated to result in higher performance than the normal-polarity case [6]. In this configuration, effective removal at very low voltages $(\sim 10 \mathrm{~V})$ was achieved due to higher currents of the discharge pulses apparently because of the field-emission properties of the CNT cathode. To evaluate the effect of the $\mathrm{SF}_{6}$ environment for reverse $\mu \mathrm{EDM}$ of CNT forests, machining tests with both the normal and reverse polarities were first conducted in a gas mixture of $50 \% \mathrm{SF}_{6}$ and $50 \% \mathrm{O}_{2}$. A $64-\mu$ m-diameter electrode was scanned along a square path of 200 $\mu \mathrm{m}$ by $200 \mu \mathrm{m}$ in a CNT forest while machining it at $25 \mathrm{~V}$ to a depth of $25 \mu \mathrm{m}$. Fig. 2 shows the structures machined at both polarities, as well as the electrode used for each case imaged after the process without cleaning. It is clear from Figs. $2 \mathrm{a}$ and $\mathrm{b}$ that the reverse-polarity process resulted in finer and sharper structures compared with the normal-polarity case, the latter exhibiting distorted shapes and rough surfaces in the machined structures. In this EDM condition at $25 \mathrm{~V}$, the reverse-polarity process was observed to generate relatively high discharge current $(\sim 16.3 \mathrm{~mA})$ that is evidently sufficient to induce desirable material removal governed by electrical discharges. In the case of the normal polarity, however, the discharge current was much lower ( $\sim 5 \mathrm{~mA})$, leading to insufficient removal by electrical discharges, therefore causing mechanical abrasion and distorted structures/surfaces. These tendencies in terms of the discharge current and machining quality are consistent with the results obtained in air [6]. 

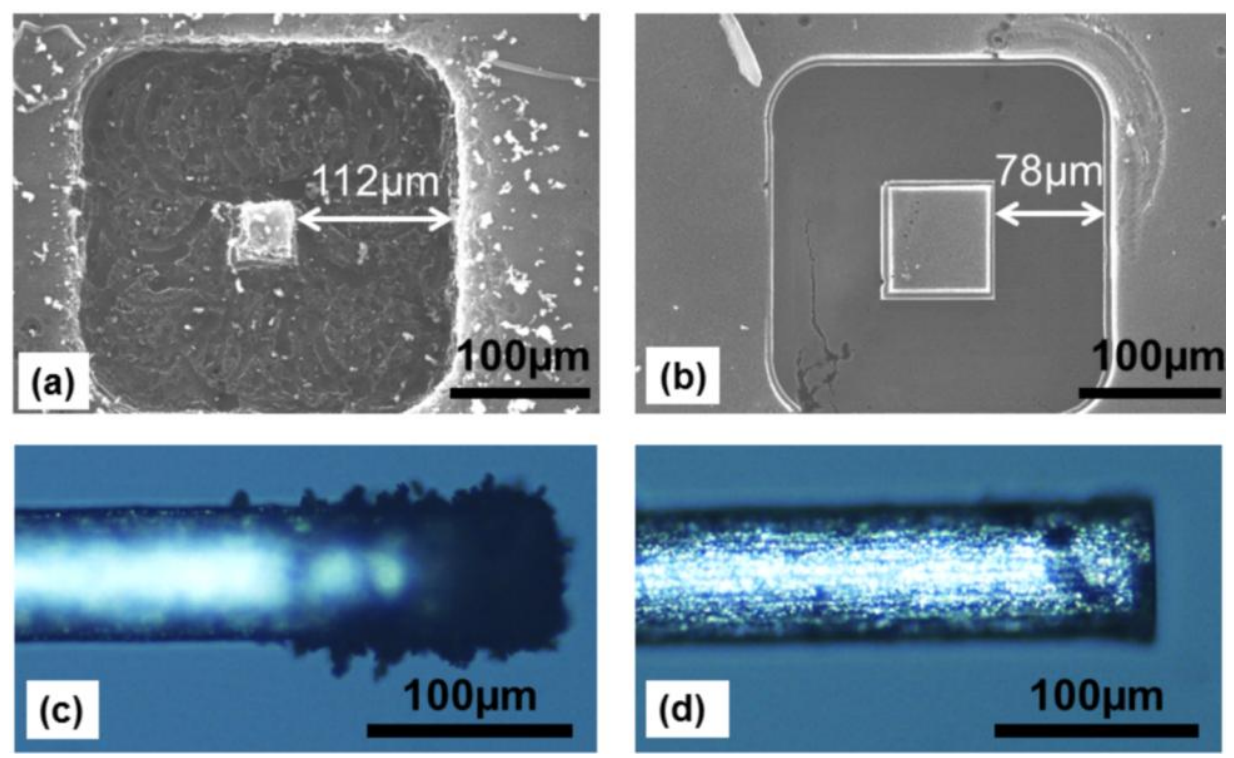

Fig. 2 - Scanning electron microscope (SEM) images of the microstructures machined in a CNT forest in $50 \% \mathrm{SF}_{6}$ and $50 \% \mathrm{O}_{2}$ at $25 \mathrm{~V}$ with (a) the normal polarity and (b) the reverse polarity. Optical images of the tungsten electrode after machining with (c) the normal polarity and (d) the reverse polarity.

As also can be seen in Fig. 2, reverse-polarity $\mu$ EDM resulted in much cleaner (less debris) structures, whereas the normal-polarity case produced more debris left on the structures and the debris accumulated on and stuck to the electrode. The debris accumulation on the electrode increases its effective diameter in a random and non-uniform manner, leading to an undesired larger gap between the walls of the resultant structures (as shown in Fig. 2a and b), thus lowering the precision in the machining process. A possible explanation for the above result may be similar to the case with reverse $\mu \mathrm{EDM}$ in air [6] - with reverse polarity, the CNT forest is the cathode and thus not subject to electron bombardment (which induces conventional thermal removal as noted earlier) in principle; therefore, CNT removal is expected to be almost entirely due to oxygen and/or fluorine plasma etching $[9,15,16]$ that decomposes CNTs into volatile 
products, forming minimal debris. With the normal-polarity condition, in contrast, the forest is the anode that is bombarded by electrons during the process and may be subject to some level of thermal removal where parts of the CNTs are melted and blown by pressure waves induced by the intense heat that the pulsed discharge produces, leaving resolidified carbon debris on the workzone and on the electrode. Based on the comparison above, the subsequent experimentations in the present study were carried out in the reverse-polarity mode, which provides more desirable results in machining precision and quality.

In order to evaluate the effect of $\mathrm{SF}_{6}$, in comparison with the conventional $\mathrm{N}_{2}$, used as the dielectric gas on reverse $\mu \mathrm{EDM}$ of CNT forests, machining tests were performed with $100 \% \mathrm{SF}_{6}$ ambient as well as with $100 \% \mathrm{~N}_{2}$ ambient. Two basic discharge phenomena in EDM should be noted before the results of the above experiment are discussed. Gas discharges can be classified into several categories based on the corresponding voltage and current values. In EDM, there are two types of discharges that most commonly occur, namely spark and arc. Spark is characterised with higher discharge voltages compare to arc. Furthermore, spark is a transient process which may ultimately lead to a continuous arc if certain electrical conditions are fulfilled. In EDM, including $\mu \mathrm{EDM}$, it is desired to have spark discharge rather than arcing, which is detrimental as it results in excessive heating that causes severe damage to the sample surfaces [17]. Fig. 3a and b shows typical discharge currents measured with $100 \% \mathrm{~N}_{2}$ and $100 \% \mathrm{SF}_{6}$, respectively. As represented in Fig. $3 \mathrm{~b}$, the $100 \% \mathrm{SF}_{6}$ case produced short pulses (pulse duration $\sim 10 \mathrm{~ns}$ ), indicating a spark-mode discharge. These pulses usually had a peak current of around $10 \mathrm{~mA}$ or less but also showed spontaneous very large peak currents (30-40 mA) occasionally. For the 
(a)

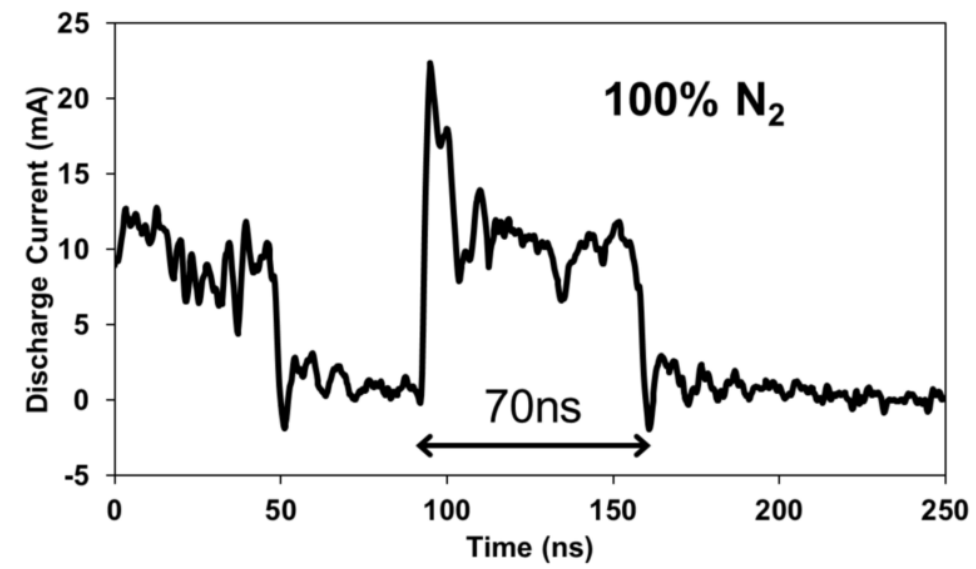

(b)

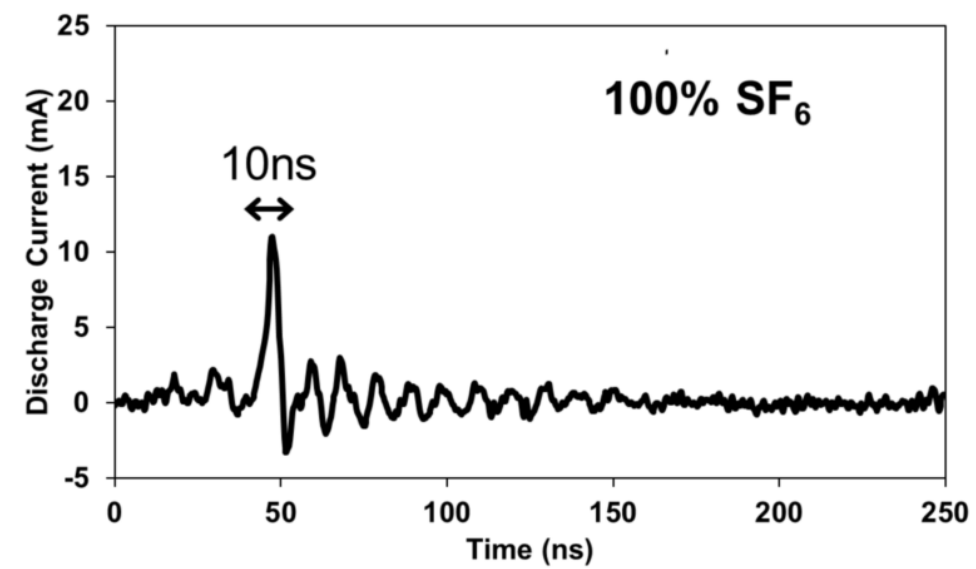

Fig. 3 - Typical measured patterns of discharge current generated at $25 \mathrm{~V}$ with the reverse polarity in (a) $100 \% \mathrm{~N}_{2}$ and (b) $100 \% \mathrm{SF}_{6}$, indicating much longer pulse duration ( $\left.70 \mathrm{~ns}\right)$ in the $\mathrm{N}_{2}$ ambient than in $\mathrm{SF}_{6}(\sim 10 \mathrm{~ns})$.

$100 \% \mathrm{~N}_{2}$ case (Fig. 3a), the process tended to produce longer pulse duration that led to frequent abnormal arcing. (These two different discharge modes, spark and arc, were also distinguishable visually through the on-machine microscope; the former case was typically observed to emit white light whereas the latter mode was with yellow-orange light emissions.) Moreover, arcing resulted in frequent short-circuit detections, preventing proper machining. Figs. $4 \mathrm{a}$ and $\mathrm{b}$ compares the results obtained in $100 \% \mathrm{~N}_{2}$ and $100 \% \mathrm{SF}_{6}$, respectively, to produce the same square patterns as those in Figs. 2a and b. Fig. 4a exhibits damage on the forest surfaces with almost no material removal. In contrast, Fig. $4 \mathrm{~b}$ shows the result of much more stable 
discharges in the form of sparks and stable removal. It has been reported in previous studies that $\mathrm{SF}_{6}$ plasma is suitable for etching of CNTs [15] and that carbon reacts with fluorine (fluorination of carbon) and forms various gaseous compounds during a plasma treatment of CNTs in an $\mathrm{SF}_{6}$ environment [16]. Although these reports did not use spark discharge, similar chemical etching
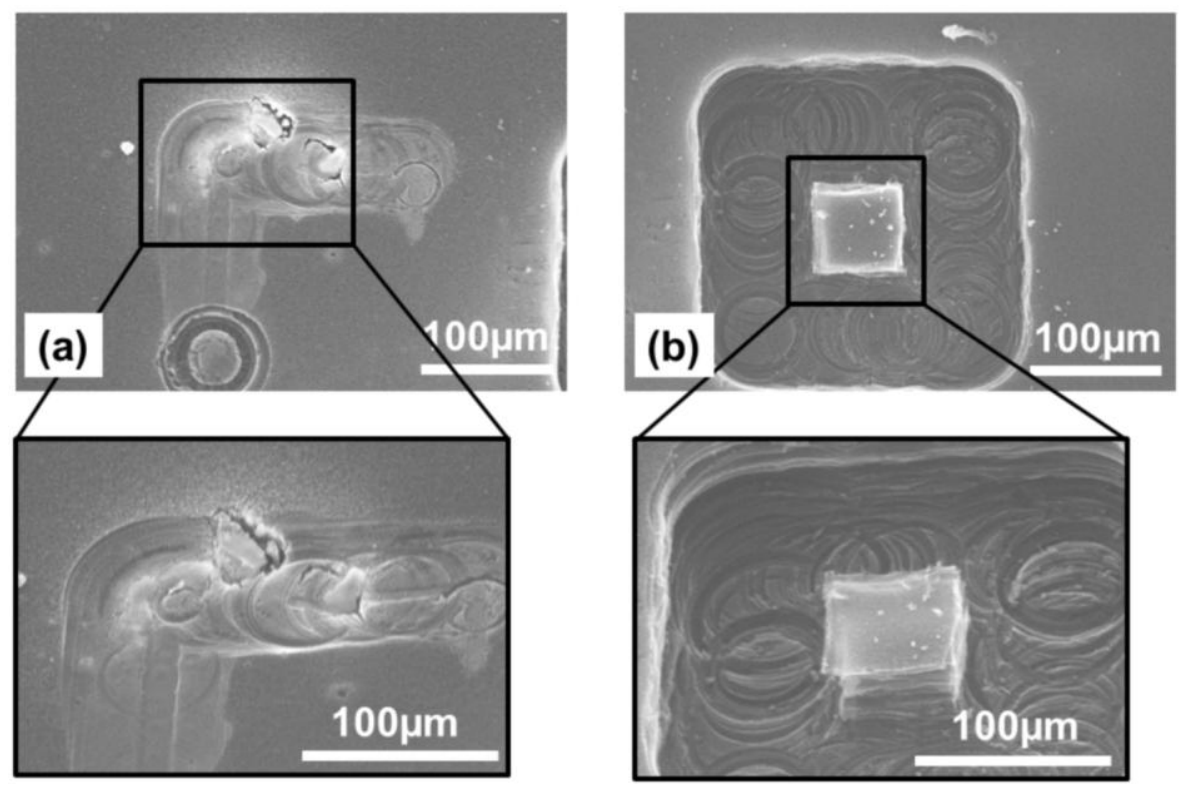

Fig. 4 - SEM images of the microstructures machined in a CNT forest at $25 \mathrm{~V}$ with the reverse polarity in (a) $100 \% \mathrm{~N}_{2}$ and (b) $100 \% \mathrm{SF}_{6}$. A close-up SEM image is also shown in each case. phenomena may occur with plasmas in the form of spark discharge, which could be the case shown in Fig. 4b. The possible reason of frequent arcing in $100 \% \mathrm{~N}_{2}$ can be understood from the fact that $\mathrm{N}_{2}$ is less electrically resistive than $\mathrm{SF}_{6}$ and thus permits the discharge gap to sustain a continuous arc between the electrode and the forest surface $[13,18,19]$.

As noted earlier, the presence of $\mathrm{O}_{2}$ in the $\mu \mathrm{EDM}$ process was reported to be essential for proper removal of CNTs in $\mathrm{N}_{2}$ ambient, in which the optimal concentration of $\mathrm{O}_{2}$ was $\sim 20 \%$ [8, 9]. To study the role of $\mathrm{O}_{2}$ in the $\mathrm{SF}_{6}$ case, the $\mu \mathrm{EDM}$ process was characterized with $\mathrm{O}_{2}$ concentrations of 10\%, 20\%, and 50\%. The results machined at $25 \mathrm{~V}$ shown in Fig. 5 suggest 
that the structural and surface quality improved with increasing $\mathrm{O}_{2}$ concentration up to $20 \%$ (Fig. $5 \mathrm{a}$ and $\mathrm{b}$ ), and that the structures became distorted (e.g., the top surface of the center post as seen in Fig. 5c, possibly due to sparks propagating and etching portions of it) again when the concentration was further increased to $50 \%$. The structure machined at $10 \mathrm{~V}$ and $20 \% \mathrm{O}_{2}$ in $\mathrm{SF}_{6}$ shown in Fig. 5d indicates deteriorated structural quality compared to Fig. 5b, the 25-V case under the same ambient. The results obtained at the same voltage levels, $25 \mathrm{~V}$ and $10 \mathrm{~V}$, with $20 \% \mathrm{O}_{2}$ in $\mathrm{N}_{2}$ ambient are shown in Fig. 5e and $\mathrm{f}$, respectively. These suggest that, in contrast to the $\mathrm{SF}_{6}-\mathrm{O}_{2}$ ambient cases, the $10-\mathrm{V}$ condition led to higher machining quality than the $25-\mathrm{V}$ condition in the $\mathrm{N}_{2}-\mathrm{O}_{2}$ ambient; this result is consistent with the previous findings reported in [6]. However, a comparison between Fig. $5 \mathrm{~b}$ and f, representing the conditions that provided the highest machining quality for the $\mathrm{SF}_{6}$ and $\mathrm{N}_{2}$ environments, respectively, suggests that $\mathrm{SF}_{6}$ results in sharper corners (without extended portions at the corner of the center post) than $\mathrm{N}_{2}$, and the sidewall of the resultant structure is smoother (free from extended layers) for $\mathrm{SF}_{6}$ ambient. Possible sources of the different optimal voltage levels for the $\mathrm{SF}_{6}$ and $\mathrm{N}_{2}$ environments (25 V and $10 \mathrm{~V}$, respectively) found above will be discussed later. 

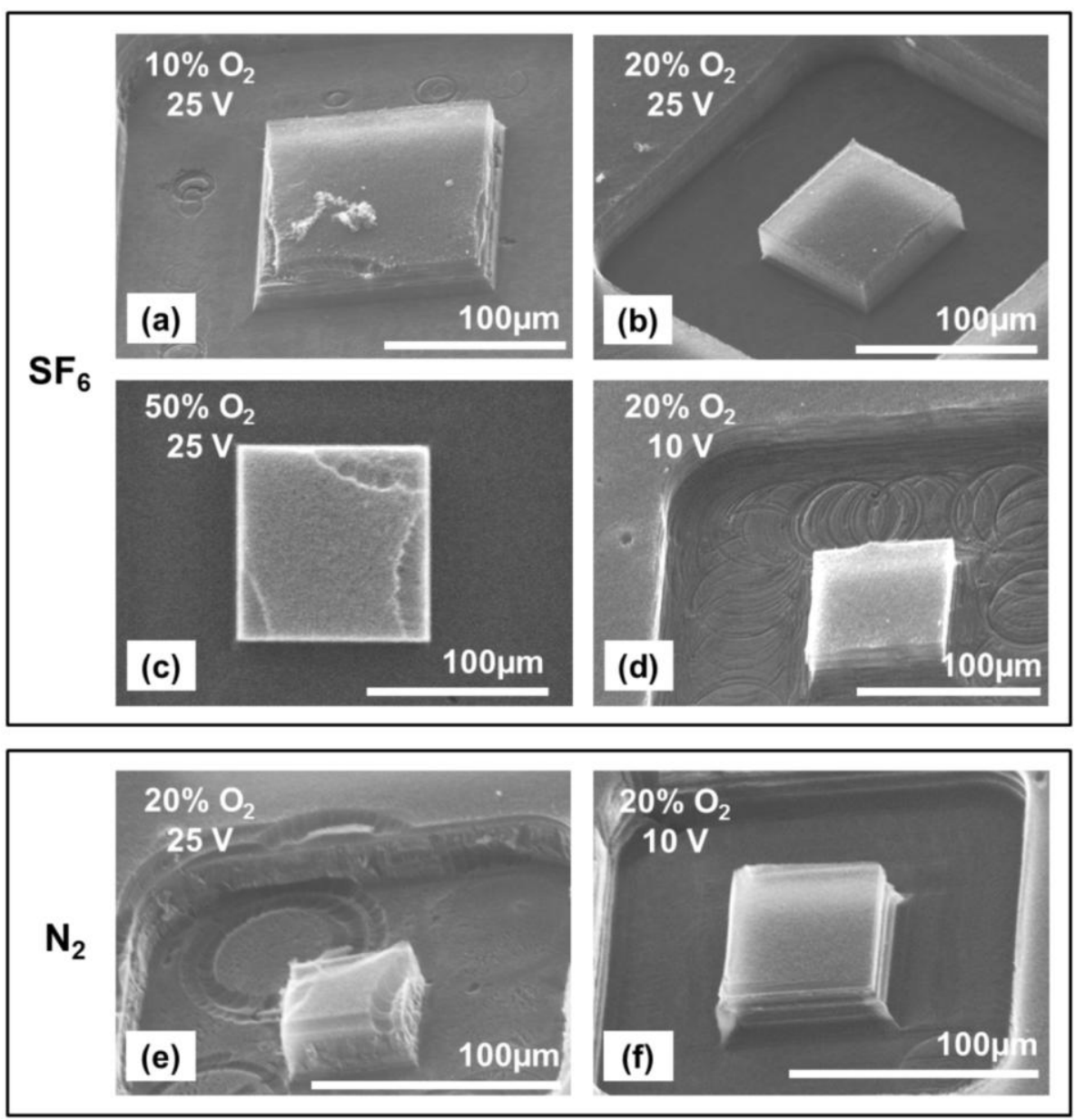

Fig. 5 - SEM images of the microstructures machined in a CNT forest: (a) $10 \% \mathrm{O}_{2}$ in $\mathrm{SF}_{6}$ at 25 $\mathrm{V}$; (b) $20 \% \mathrm{O}_{2}$ in $\mathrm{SF}_{6}$ at $25 \mathrm{~V}$; (c) $50 \% \mathrm{O}_{2}$ in $\mathrm{SF}_{6}$ at $25 \mathrm{~V}$; (d) $20 \% \mathrm{O}_{2}$ in $\mathrm{SF}_{6}$ at $10 \mathrm{~V}$; (e) $20 \% \mathrm{O}_{2}$ in $\mathrm{N}_{2}$ at $25 \mathrm{~V}$; (f) $20 \% \mathrm{O}_{2}$ in $\mathrm{N}_{2}$ at $10 \mathrm{~V}$.

Fig. 6a shows the measured values of the average peak discharge current (calculated from 300 individual discharge pulses) and of the discharge gap as a function of $\mathrm{O}_{2}$ concentration in $\mathrm{SF}_{6}$. The discharge gap was calculated as the half of the dimensional difference between the measured width of a groove machined in a forest and the diameter of the electrode used. Fig. $6 \mathrm{~b}$ compares the discharge gaps measured in the structures obtained with the $\mathrm{SF}_{6}$ and $\mathrm{N}_{2}$ environments (both at $20 \% \mathrm{O}_{2}$ ) at the two discharge voltages of $25 \mathrm{~V}$ and $10 \mathrm{~V}$. Fig. 7 shows the 
(a)

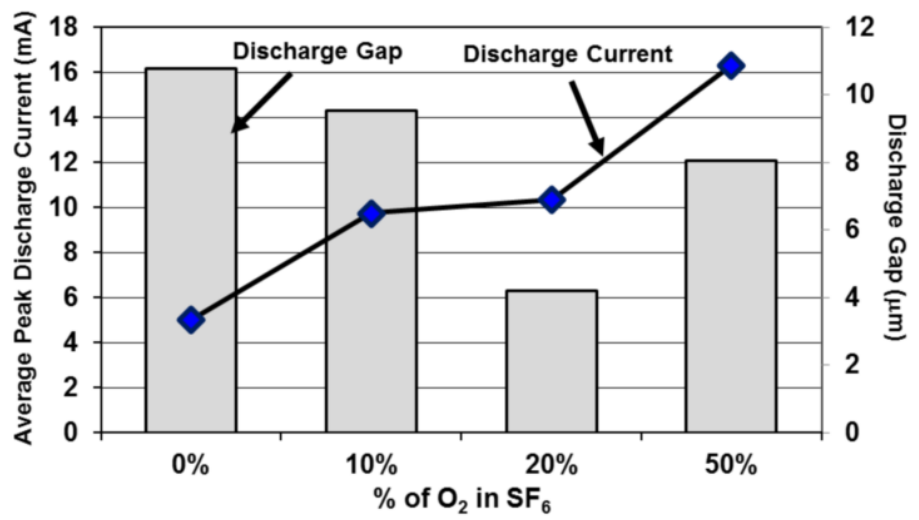

(b)

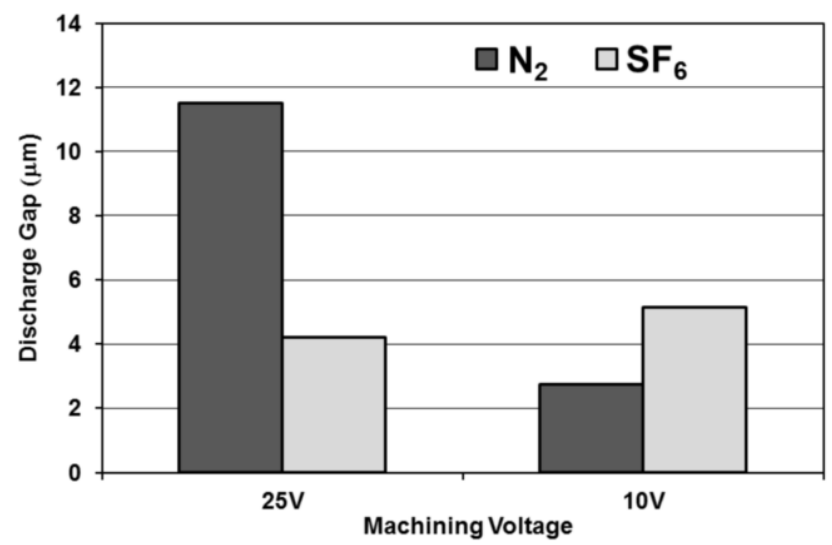

Fig. 6 - (a) Average peak discharge current generated at $25 \mathrm{~V}$ and resultant discharge gap with different $\mathrm{O}_{2}$ concentrations in $\mathrm{SF}_{6}$. (b) Measured discharge gaps resulted from the $\mathrm{SF}_{6}$ and $\mathrm{N}_{2}$ environments (with $20 \% \mathrm{O}_{2}$ ) and two different discharge voltages.

measured $\mathrm{Z}$ position of the electrode captured during machining processes with different gas compositions and discharge voltages. The ripples seen in Fig. 7 were caused by the retraction motion of the $\mathrm{Z}$ stage due to the short-circuit events occurred in the processes as noted earlier. As shown in Fig. 6a, the discharge current was observed to have an increasing trend with $\mathrm{O}_{2}$ concentration in $\mathrm{SF}_{6}$ at $25 \mathrm{~V}$; however, the discharge gap exhibited the minimal value (of 4.2 $\mu \mathrm{m})$ at $20 \% \mathrm{O}_{2}$. These results may be explained as follows: The $\mathrm{O}_{2}$ concentration at $10 \%$ caused frequent short-circuit detections as shown in Fig. 7, which provided more energy for plasma etching noted earlier, resulting in a larger gap. For the $100 \% \mathrm{SF}_{6}\left(\mathrm{O}_{2}\right.$ free $)$ case, although the 
machining was observed to be smooth (with little short-circuit detection as shown in Fig. 7) with a low average peak current $(\sim 5 \mathrm{~mA})$, the very large pulses (with peaks of $30-40 \mathrm{~mA}$ ) occasionally observed in this $100 \% \mathrm{SF}_{6}$ condition discussed earlier may have caused larger removal and discharge gap. For an $\mathrm{O}_{2}$ concentration above 20\%, the process generated short pulses with higher peak currents in a consistent manner, which also led to a larger discharge gap.

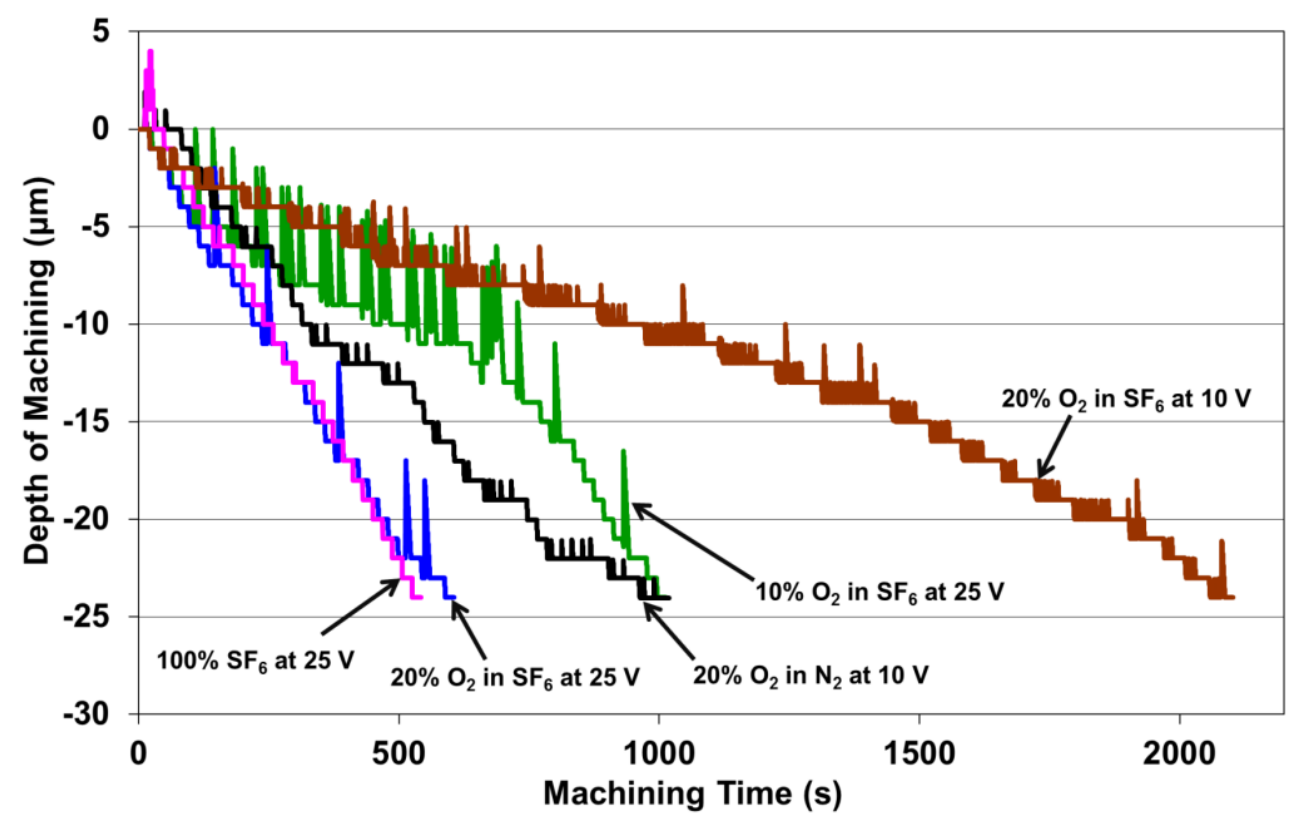

Fig. 7 - Electrode's position along the $\mathrm{Z}$ axis with machining time for different gas media and EDM conditions measured during patterning shown in Fig. 5. The retracting distance upon a short-circuit detection was set to $5 \mu \mathrm{m}$ for all the cases except for the conditions $20 \% \mathrm{O}_{2}$ in $\mathrm{N}_{2}$ and $\mathrm{SF}_{6}$ at $10 \mathrm{~V}$, in which the length was set to $1 \mu \mathrm{m}$.

As discussed above and shown in Fig. 6 b, in $\mathrm{SF}_{6}\left(\right.$ at $\left.20 \% \mathrm{O}_{2}\right)$, processing at $25 \mathrm{~V}$ resulted in the minimum discharge gap and the highest machining quality; however, this is not the case for $\mathrm{N}_{2}$ (at the same $\mathrm{O}_{2}$ concentration), in which processing at $10 \mathrm{~V}$ led to the minimum gap and the 
highest machining quality. In the $\mathrm{SF}_{6}$ environment, $25 \mathrm{~V}$ was a suitable voltage level to produce spark discharge pulses and perform smooth machining (Fig. 5b); however, $10 \mathrm{~V}$ may have made the discharge gap too small because of the high-dielectric ambient and thus caused physical touching and mechanical rubbing between the rotating electrode and the forest surface (which may have caused the circular marks on the bottom of the structure shown in Fig. 5d due to nonideal mechanical/positioning instability in the $\mu \mathrm{EDM}$ system used, deteriorating the processed structure/surfaces. This undesired mechanical contact may have also occurred on the sidewalls of the patterned structures and caused slight bending or displacement of the CNTs on the walls, which may be the probable cause of the larger gap compared with the $\mathrm{N}_{2}$ environment case at 10 $\mathrm{V}$ (Fig. 6b) and of the very frequent short circuits or long machining time (Fig. 7). In the $\mathrm{N}_{2}$ environment, on the contrary, the tendency of uncontrolled large spark and/or arcing (similar to the $100 \% \mathrm{~N}_{2}$ case) was evident when $25 \mathrm{~V}$ was used. This is believed to be a major source of the structural distortion observed (Fig. 5e) as well as the enlarged gap (Fig. 6b). Lowering the voltage to $10 \mathrm{~V}$ improved the removal quality (Fig. 5f) while decreasing the discharge gap. However, these favourable features at $10 \mathrm{~V}$ in the $\mathrm{N}_{2}$ case come with the price of machining stability and efficiency - a relatively high rate of short circuiting, presumably due to the low discharge energy causing insufficient removal, was observed to slow the process at this $10-\mathrm{V}$ condition (e.g., Fig. 7 shows that the removal at $10 \mathrm{~V}$ in $\mathrm{N}_{2}$ was $\sim 1.7 \times$ slower than the case at 25 $\mathrm{V}$ in the $\mathrm{SF}_{6}$ environment). This high rate of short circuiting may have also introduced undulation of the side walls of the resultant structure as shown in Fig. $5 \mathrm{f}$.

The elements on the surfaces of the forest microstructures (the bottom of the trenches) reverse- $\mu \mathrm{EDMed}$ in the $\mathrm{SF}_{6}-\mathrm{O}_{2}$ ambient were characterized using energy-dispersive $\mathrm{X}$-ray spectroscopy (EDX) at 20-keV beam voltage (the beam spot size was $\sim 2 \mu \mathrm{m}$, almost $40 \times$ smaller 
than the width of the trenches, which ensured that the EDX data were obtained from the bottom of the trenches). The results from the surfaces processed with $20 \%$ and $10 \% \mathrm{O}_{2}$ (Fig. 8) indicate insignificant detectable sulphur and fluorine in both cases, suggesting that the use of $\mathrm{SF}_{6}$ does
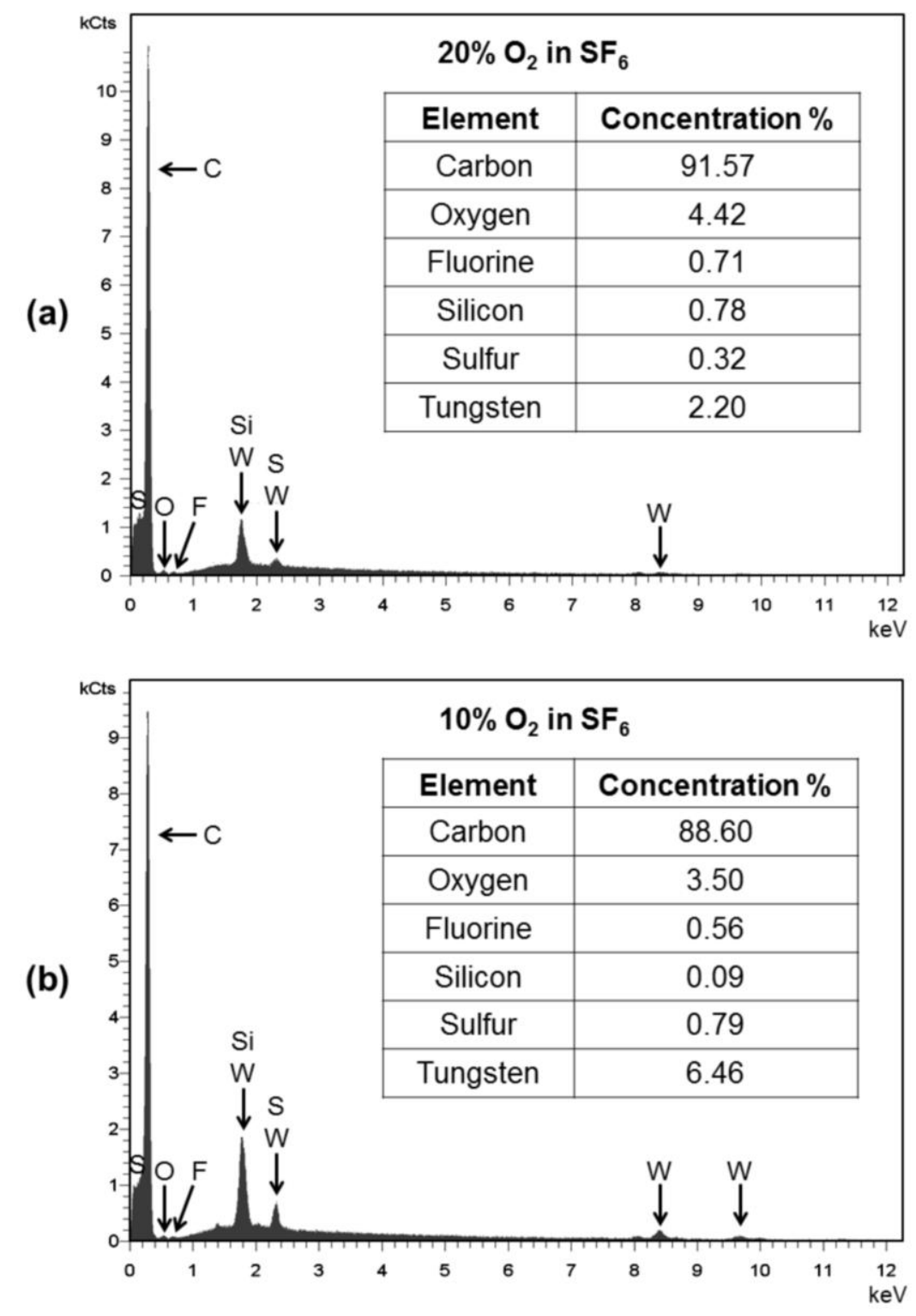

Fig. 8 - EDX analysis results for the CNT-forest surfaces machined in $\mathrm{SF}_{6}$ with (a) $20 \% \mathrm{O}_{2}$ and (b) $10 \% \mathrm{O}_{2}$. 
not cause any considerable contamination caused by the ambient gas itself. A low level of silicon detected is most likely due to the presence of the silicon substrate below the forest. The results also show that the level of tungsten, the contaminant due to wear of the electrode, is much lower at $20 \% \mathrm{O}_{2}$ than the $10 \% \mathrm{O}_{2}$ case, a favourable characteristic that the process at the optimal $\mathrm{O}_{2}$ concentration provides. Interestingly, it can be seen that the level of silicon is substantially reduced (by a factor of $\sim 9$ ) with the lower $\mathrm{O}_{2}$ concentration of $10 \%$. A potential reason behind this could be as follows; at $10 \% \mathrm{O}_{2}$, the tungsten level is almost $3 \times$ higher (6.46\% as indicated), which may form a thin layer on the machined surface and reduce the electron beam penetration, as well as the escape of the generated X-rays from underneath, leading to less detection of silicon. Another fact that is worth noting is that iron was not detected in the machined surfaces using both $\mathrm{O}_{2}$ concentrations. This result could be related to the following two possibilities. One is that the CNTs may be predominantly root grown, thus iron remains on the substrate surface that is too far from the probed forest surfaces to be detected, given its small amount (as can be seen, even silicon shows up with very small signals, although the substrate is bulk silicon). The other is that iron may be originally present on the forest surface (due to potential tip growth of CNTs) but removed by the $\mu \mathrm{EDM}$ process. As regards the tungsten level in the $\mathrm{N}_{2}$ environment, previous report shows $1.25 \%$ of tungsten contamination under the condition corresponding to the optimal $\mathrm{N}_{2}$ case [20]. The tungsten level observed in the current study for $\mathrm{SF}_{6}(2.2 \%$, Fig. 8a) is somewhat larger than the above level; however the electrode's (tungsten's) consumption condition can be affected by not only the gas medium but also the electrical contact (contact resistance) to the forest which can vary from sample to sample and may lead to different values in tungsten contamination as stated above. 


\section{Conclusions}

The effect of using $\mathrm{SF}_{6}$, a high dielectric-strength gaseous medium, mixed with $\mathrm{O}_{2}$, a chemical decomposition agent, in dry $\mu \mathrm{EDM}$ of pure CNT forests has been investigated. The characterization was performed for both normal and reverse polarities in the $\mu$ EDM process. The reverse-polarity condition was found to increase the discharge current, possibly because of superior field-emission properties of CNTs that served as the cathode in the reverse condition. It was found that, in contrast to EDM in $\mathrm{N}_{2}$, the process could be performed in $\mathrm{SF}_{6}$ only, without the need for $\mathrm{O}_{2}$, while the addition of $\mathrm{O}_{2}$ in $\mathrm{SF}_{6}$ improved the machining precision/quality. It was demonstrated that reverse $\mu \mathrm{EDM}$ in the new gaseous system was effective in lowering the machining voltage, leading to finer and cleaner CNT removal compared to the normal-polarity condition. Moreover, with a discharge voltage of $25 \mathrm{~V}$ and an $\mathrm{O}_{2}$ concentration of $20 \%$, the use of $\mathrm{SF}_{6}$ was revealed to give better results compared to $\mathrm{N}_{2}$, the conventional ambient medium; the discharge gap could be reduced to $4.2 \mu \mathrm{m}$. At a lower voltage level (10 V), an $\mathrm{N}_{2}$ ambient with $20 \% \mathrm{O}_{2}$ produced an even smaller discharge gap, although the occurrence of short circuits slowed the removal process somewhat. The $\mathrm{SF}_{6}-\mathrm{O}_{2}$ gaseous system at $25 \mathrm{~V}$ was demonstrated to enable not only more stable and faster processing but also relatively higher machining quality compared to the optimal $\mathrm{N}_{2}-\mathrm{O}_{2}$ ambient case at $10 \mathrm{~V}$. EDX analysis revealed that reverse $\mu$ EDM in the optimal $\mathrm{SF}_{6}-\mathrm{O}_{2}$ ambient leaves more than $90 \%$ carbon in the etched region with a minimal amount of the electrode element or the gas elements. 
Acknowledgments

The authors thank Mohamed Sultan Mohamed Ali for his assistance in the use of the $\mu \mathrm{EDM}$ system. We also thank Mehran Vahdani Moghaddam for assistance with the nanotube growth process. This work was partially supported by the Natural Sciences and Engineering Research Council of Canada, the Canada Foundation for Innovation, the British Columbia Knowledge Development Fund, and the BCFRST Foundation/British Columbia Innovation Council. K. Takahata is supported by the Canada Research Chairs program.

\section{REFERENCES}

[1] Treacy MMJ, Ebbesen TW, Gibson JM. Exceptionally high Young's modulus observed for individual carbon nanotubes. Nature 1996; 381:678-80.

[2] Avouris P, Chen J. Nanotube electronics and optoelectronics. Mater Today, 2006; 9:46-54.

[3] Hamada N, Sawada S, Oshiyama A. New one-dimensional conductors: Graphitic microtubules. Phys Rev Lett 1992; 68:1579 -81.

[4] Hsieh K, Tsai T, Wan D, Chen H, Tai N. Iridescence of patterned carbon nanotube forests on flexible substrates: from darkest materials to colorful films. ACS Nano 2010; 4:1327-36.

[5] Hone J, Llaguno MC, Biercuk MJ, Johnson AT, Batlogg B, Benes Z, et al. Thermal properties of carbon nanotubes and nanotube-based materials. Appl Phys A 2002; 74:33943.

[6] Saleh T, Dahmardeh M, Bsoul A, Nojeh A, Takahata K. Field-emission-assisted approach to dry micro-electro-discharge machining of carbon-nanotube forests. J Appl Phys 2011; 110:103305. 
[7] Khalid W, Mohamed Ali MS, Dahmardeh M, Choi Y, Yaghoobi P, Nojeh A, et.al. Highaspect-ratio, free-form patterning of carbon nanotube forests using micro-electro-discharge machining,. Diam Relat Mater 2010; 19:1405-10.

[8] Dahmardeh M, Khalid W, Mohamed Ali MS, Choi Y, Yaghoobi P, Nojeh A, et.al. Highaspect-ratio, 3-D micromachining of carbon-nanotube forests by micro-electro-discharge machining in air. 24th IEEE Int'1 Conf. Micro Electro Mechanical Systems (MEMS 2011), Cancun, Mexico, 2011; p. 272-5.

[9] Dahmardeh M, Nojeh A, Takahata K. Possible mechanism in dry micro-electro-discharge machining of carbon-nanotube forests: A study of the effect of oxygen. J Appl Phys 2011; 109:093308.

[10] Zhu YW, Sow CH, Sim MC, Sharma G, Kripesh V. Scanning localized arc discharge lithography for the fabrication of microstructures made of carbon nanotubes. Nanotechnology 2007; 18:385304.

[11] Ok JG, Kim BH, Chung DK, Sung WY, Lee SM, Lee SW, et al. Electrical discharge machining of carbon nanomaterials in air: Machining characteristics and the advanced field emission applications. J Micromech Microeng 2008; 18:025007.

[12] Liang B, Ogino A, Nagatsu M. Discharge characteristics of a nano-sized electrode with aligned carbon nanotubes grown on a tungsten whisker tip under various gas conditions. J Phys D Appl Phys 2010; 43:275202.

[13] Rokunohe T, Yagihashi Y, Endo F, Oomori T. Fundamental insulation characteristics of air, $\mathrm{N}_{2}, \mathrm{CO}_{2}, \mathrm{~N}_{2} / \mathrm{O}_{2}$, and $\mathrm{SF}_{6} / \mathrm{N}_{2}$ mixed gases. Elect Eng Jpn 2006; 155:619-25.

[14] Jahan MP, Wong YS, Rahman M. A study on the quality micro-hole machining of tungsten carbide by micro-EDM process using transistor and RC-type pulse generator. J Mater 
Process Tech 2009; 209:1706-16.

[15] Hou Z, Cai B, Liu H, Xu D. Ar, $\mathrm{O}_{2}, \mathrm{CHF}_{3}$, and $\mathrm{SF}_{6}$ plasma treatments of screen-printed carbon nanotube films for electrode applications. Carbon 2008; 46 :405-13.

[16] Barlow A, Birch A, Deslandes A, Quinton JS. Plasma fluorination of highly ordered pyrolytic graphite and single walled carbon nanotube surfaces. 2006 Int'l Conf. Nanoscience and Nanotechnology (ICONN 2006), Brisbane, Australia, 2006; p. 103-6.

[17] Descoeudres A. Characterization of electrical discharge machining plasmas. Lausanne, Switzerland, École Polytechnique Fédérale de Lausanne, PhD Thesis, 2006.

[18] Geballe R, Reeves ML. A condition on uniform field breakdown in electron-attaching gases. Phys Rev 1953; 92:867-8.

[19] Nitta T, Shibuya Y. Electrical breakdown of long gaps in sulfur hexafluoride. IEEE Trans Power Apparatus Syst 1971; 90:1065-71.

[20] Saleh T, Dahmardeh M, Bsoul A, Nojeh A, Takahata K. High precision dry micro-electrodischarge machining of carbon-nanotube forests with ultralow discharge energy. 25th IEEE Int'1 Conf. Micro Electro Mechanical Systems (MEMS 2012), Paris, France, 2012; p. 25962. 